\title{
COUNCIL AND BOARD OF TRUSTEES
}

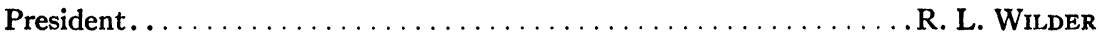
Ex-President $\ldots \ldots \ldots \ldots \ldots \ldots \ldots \ldots \ldots \ldots \ldots \ldots \ldots \ldots \ldots \ldots \ldots, T$. WHYBURN

f. V. AHLFORS EMIL ARTIN

ANTONI ZYGMUND

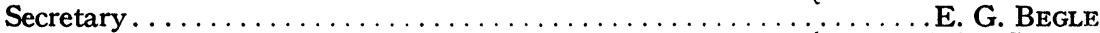

Associate Secretaries......................... $\left\{\begin{array}{r}\text { J. W. GREEN }{ }^{1} \\ \text { J. H. RoBERTS }\end{array}\right.$

R. D. SCHAFER

J. W. T. Youngs

Treasurer

Bulletin Editorial Committee.

W. T. MARTIN

G. B. PRICE

Proceedings Editorial Committee

RICHARD BRAUER SHIzUO KaKUTANI

A. C. Schaeffer

L. V. AHLFORS

Transactions and Memoirs Editorial Committee......... Herbert BUSEMANN ${ }^{2}$

J. L. DooB

SAMUEL EILENBERg

A. A. Albert

Colloquium Editorial Committee.................

DEANE MONTGOMERY

Mathematical Reviews Editorial Committee

R. P. BOAS, JR. J. L. DOoB

EINAR HILLE

Mathematical Surveys Editorial Committee.

Committee on Printing and Publishing.

MaX SHIFFMaN

R. J. WALKER LEO ZIPPIN

Representatives on Board of Editors of the American Journal of Mathematics.

E. F. BECKENBACH C. J. ReEs

\section{REINHOLD BAER}

ANDRE WEIL

Members at LaRge
To serve until
December, 1955
F. B. JONES
E. E. MoIse
B. J. Petris
R. M. Thrall
G. W. WhitehEAD

To serve until

December, 1957

E. F. BECKENBACH

David BlackWELL.

R. H. Fox

K. O. FRIEDRICHS

G. DE B. RobINSON

\section{BOARD OF TRUSTEES}
H. F. Bohnenblust
Einar Hille
G. A. HedLund

$$
\text { A. E. MEDER, JR., ex officio }
$$
Deane MONTgOMery
MINA S. REES
R. L. WILDER, ex officio

\footnotetext{
1 Replaced by V. L. Klee, Jr. on May 1, 1955.

Replaced by Leo Zippin for academic year 1954-1955.

- Replaced by A. W. Tucker on August 1, 1955.
} 